\title{
CALIBRATION OF AN ELECTRONIC PHONOCARDIOGRAPH
}

\author{
BY \\ A. W. SLOAN* AND J. R. GREER \\ From the Departments of Physiology and of Natural Philosophy, University of Glasgow \\ Received August 28, 1954
}

Calibration of a phonocardiograph is more difficult than calibration of an electrocardiograph and the clinical value of the procedure is less obvious. The loudness of the heart sounds and murmurs at the front of the chest does not depend exclusively on cardiac activity but is influenced also by the position of the heart in the chest, by the nature and thickness of the chest wall, and by extraneous factors related to disease of the lungs, pleura, or pericardium. For a valid comparison of phonocardiographic with auscultatory findings it is, however, essential to have some indication on the phonocardiogram of the absolute loudness of heart sounds and murmurs at the chest wall.

In logarithmic phonocardiography (Rappaport and Sprague, 1942) the low-frequency vibrations are selectively attenuated as they are in auscultation by the human auditory mechanism: the amplitude of the deflections on a logarithmic phonocardiogram are therefore directly related to the loudness of the audible sounds. In linear phonocardiography, without this low-frequency attenuation, the audio-frequency heart sound vibrations are masked on the record by lower-frequency vibrations of much greater amplitude: the deflections on a linear phonocardiogram thus correspond to palpable pulsation or thrills rather than to auditory phenomena. In stethoscopic phonocardiography an attempt is made to include the distortion of sound normally introduced by a stethoscope, without the distortion due in auscultation to the human ear. Because the stethoscopic phonocardiogram is an arbitrary recording, not corresponding to any sensory phenomenon, we do not favour it.

\section{METHODS}

The earliest effective attempt at calibrated phonocardiography was that of Mannheimer (1940, 1941), who recorded sound phenomena in six frequency ranges. Mannheimer calibrated his own microphone, using the piston-phone technique described by Olson and Massa (1934).

For the study of basal diastolic murmurs, Wells et al. (1949) recorded at the end of each phonocardiogram a signal corresponding to a standard sound at 500 cycles per second and 80 decibels above an arbitrary threshold of audibility. Counihan et al. (1951), for the investigation of lowfrequency præcordial vibrations, increased the standard signal to 90 decibels above this threshold. Dunn and Rahm (1952) calibrated their microphones with a piston-phone. Besterman and Harrison (1953) recorded a 50-cycle signal.

We have designed a calibration device for use with an electronic phonocardiograph already described in this Journal (Campbell et al., 1952). A similar device could be fitted to the input stage of any other electronic phonocardiograph.

Choice of Calibration Signal. A standard frequency of 50 cycles per second was chosen because our investigations have been of heart sounds rather than of murmurs; for the latter a higher frequency might be preferable but this would involve a much more complex calibration device. We believe that described here to be suitable for general purpose phonocardiography.

* Present address: Physiology Department, University of Cape Town. 
According to Fletcher (1948) the auditory threshold at 50 cycles per second is 0.35 dynes per sq. $\mathrm{cm}$. : according to Wood (1940) it is 0.30 dynes per sq. $\mathrm{cm}$. Our arbitrary threshold $(0.33$ dynes per sq. $\mathrm{cm}$.) approximates to the mean of these: our standard signal, 25 decibels above this threshold, represents a sound which would be clearly audible under normal clinical conditions.

Present Instrument. Our microphone has been calibrated by the National Physical Laboratory: from their calibration we know that our standard signal corresponds to an output of $2.4 \mathrm{mV}$ from the particular crystal microphone used for this investigation. The validity of this calibration, of course, depends on constant sensitivity of the microphone. Dunn and Rahm $(1953 a, b)$ found that the sensitivity of a piezo-electric crystal microphone remains very constant for at least two years.

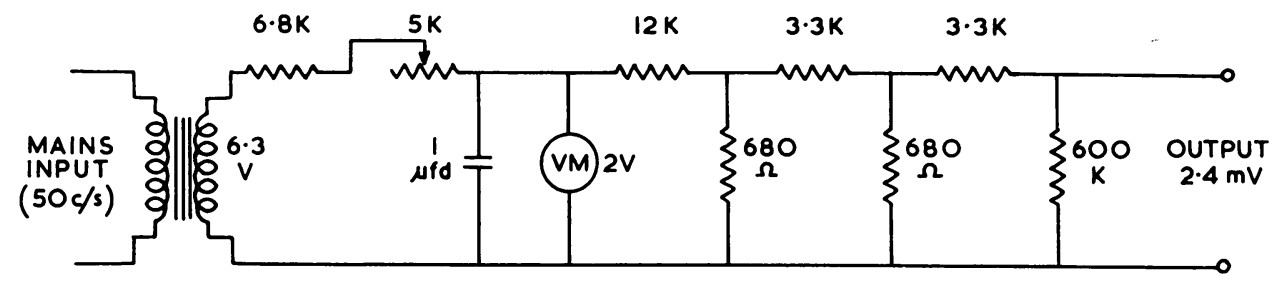

FIG. 1.-Circuit of calibrator unit. Resistor values in $\Omega$ (ohms) and $\mathrm{K}\left(10^{3} \mathrm{ohms}\right) . \quad \mathrm{V}$, volts; $\mathrm{mV}$, millivolts; VM, voltmeter.

Fig. 1 shows the circuit of the device. The 50 cycles/sec. mains supply is stepped down by a filament transformer to approximately $6.3 \mathrm{~V}$, and is then further reduced and set to exactly $2.0 \mathrm{~V}$ by a variable resistor and voltmeter, both mounted on the front panel. This allows compensation to be made for fluctuations in mains voltage. A capacitor is mounted across the voltmeter to form, with the voltage-dropping resistors, a simple resistance-capacitor filter, which eliminates highfrequency interference and discriminates against harmonic distortion in the incoming wave-form. With a linear amplifier this distortion would not be significant but, when logarithmic filters are inserted, the harmonic content of the lower frequencies is greatly emphasized; this excessive distortion would affect the apparent size of the calibration envelope.

A three-stage $600 \Omega$ ladder network of high-stability resistors reduces the $2.0 \mathrm{~V}$ input to an injection level of $2.4 \mathrm{mV}$. Under test an input increased to $20.0 \mathrm{~V}$ gave an output of $24.0 \mathrm{mV}$, which was large enough for accurate measurement on an oscilloscope.

\section{RESULTS}

Fig. 2 shows logarithmic and linear phonocardiograms from a normal subject. For the logarithmic records the gain is high but the standard signal, being of low frequency, is attenuated by the filters in the preamplifier. For linear phonocardiography the gain is much reduced and the filters are out of circuit; at 50 cycles per second these effects tend to cancel out, so the standard signal is of the same order of amplitude on both logarithmic and linear records.

Fig. 3, from a patient with patent ductus arteriosus, shows how the calibrated record gives an indication of the loudness of the sounds and murmurs. The logarithmic phonocardiogram from the mitral area shows a systolic murmur of low amplitude, although the calibration signal is quite large, showing high gain on the instrument. At the pulmonary area the murmur is of greater amplitude in spite of a lower gain setting, shown by the small calibration signal.

Fig. 4 is a logarithmic phonocardiogram from a patient with mitral stenosis: this was recorded at low gain because of the loud heart sounds and an audible mid and late diastolic murmur fails to appear on the record. Fig. 5 is from a patient with advanced pulmonary emphysema: by reference to the standard signal the sounds, recorded at high gain, are seen to be faint. 


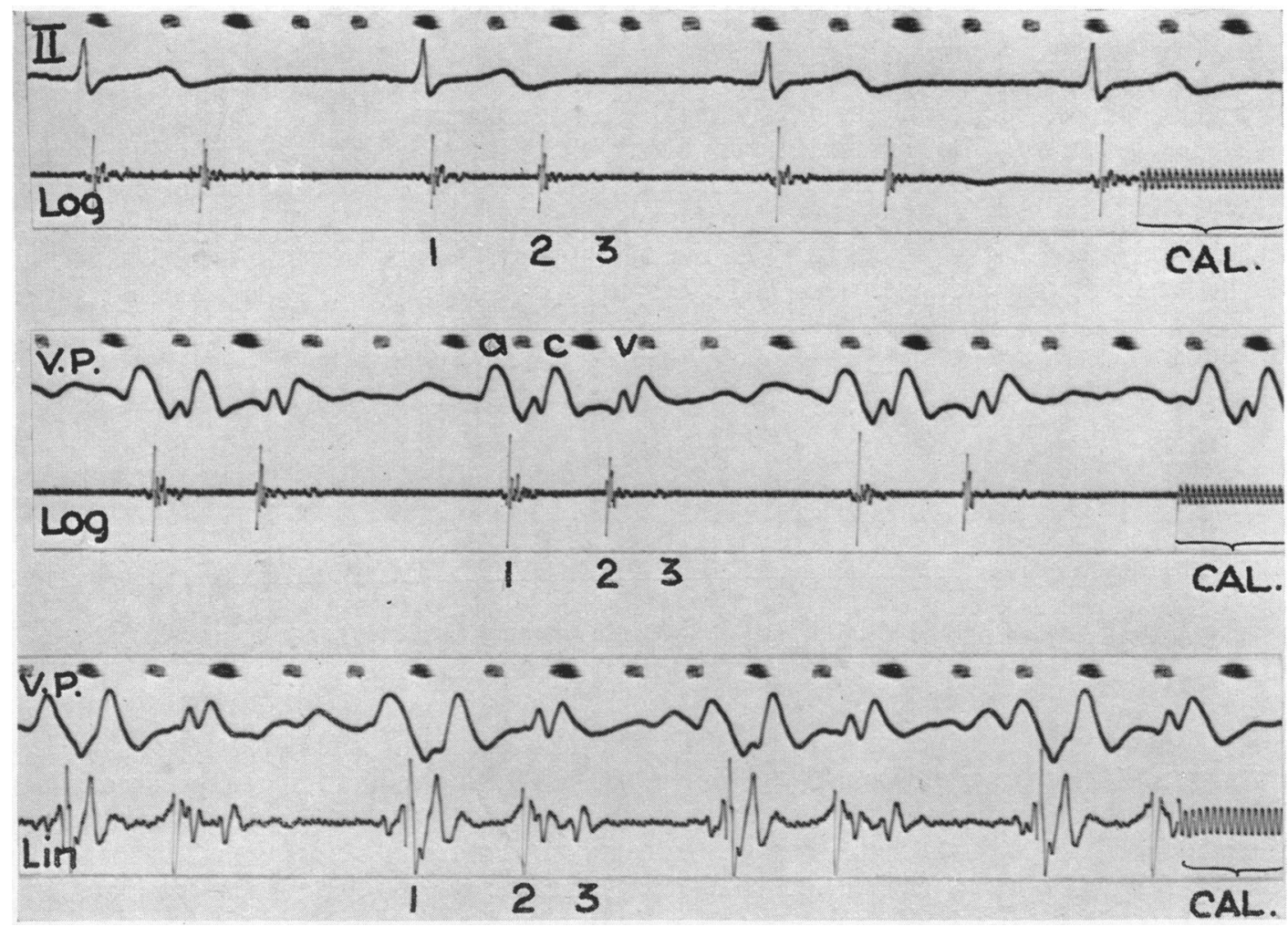

FIG. 2.-Calibrated phonocardiograms from the mitral area of a healthy subject. II, electrocardiogram (lead II); V.P., jugular venous pulse; a,c,v, waves of venous pulse; Log, logarithmic phonocardiogram; Lin, linear phonocardiogram; 1,2,3, heart sounds; CAL, calibration signal. In this and in subsequent records the calibration signal corresponds to a sound at 50 cycles per second exerting a pressure of 5.9 dynes per sq. $\mathrm{cm}$. on the microphone; this is 25 decibels above the threshold of audibility. The time markings at the top of this and subsequent records are at $1 / 5 \mathrm{sec}$. intervals.

\section{DiscusSION}

Clinically it is impracticable to run a phonocardiograph at constant gain, as is usual with an electrocardiograph. Since the amplitude of the loudest heart sounds is of the order of forty times that of the faintest ones that can be heard (Williams and Dodge, 1926) it is necessary to adjust the gain of the instrument to the loudness of the sounds in order to record the vibrations on a narrow strip of photographic material: this may bring up on the record a heart sound or murmur, which is inaudible, or may eliminate from the record an audible phenomenon. This discrepancy between auscultatory and phonocardiographic findings is inevitable with most phonocardiographs, but the situation is clarified if a standard signal is recorded after the phonocardiogram. The amplitude of the deflections of the standard signal depends on the existing gain and filter settings, and the absolute loudness of heart sounds and murmurs may be calculated by reference to an appropriate signal.

\section{SUMmary}

A simple calibration device is described, which may be applied to the input stage of an electronic phonocardiograph. A standard signal, corresponding to a pure tone at $50 \mathrm{c} / \mathrm{s}$ and 25 decibels 


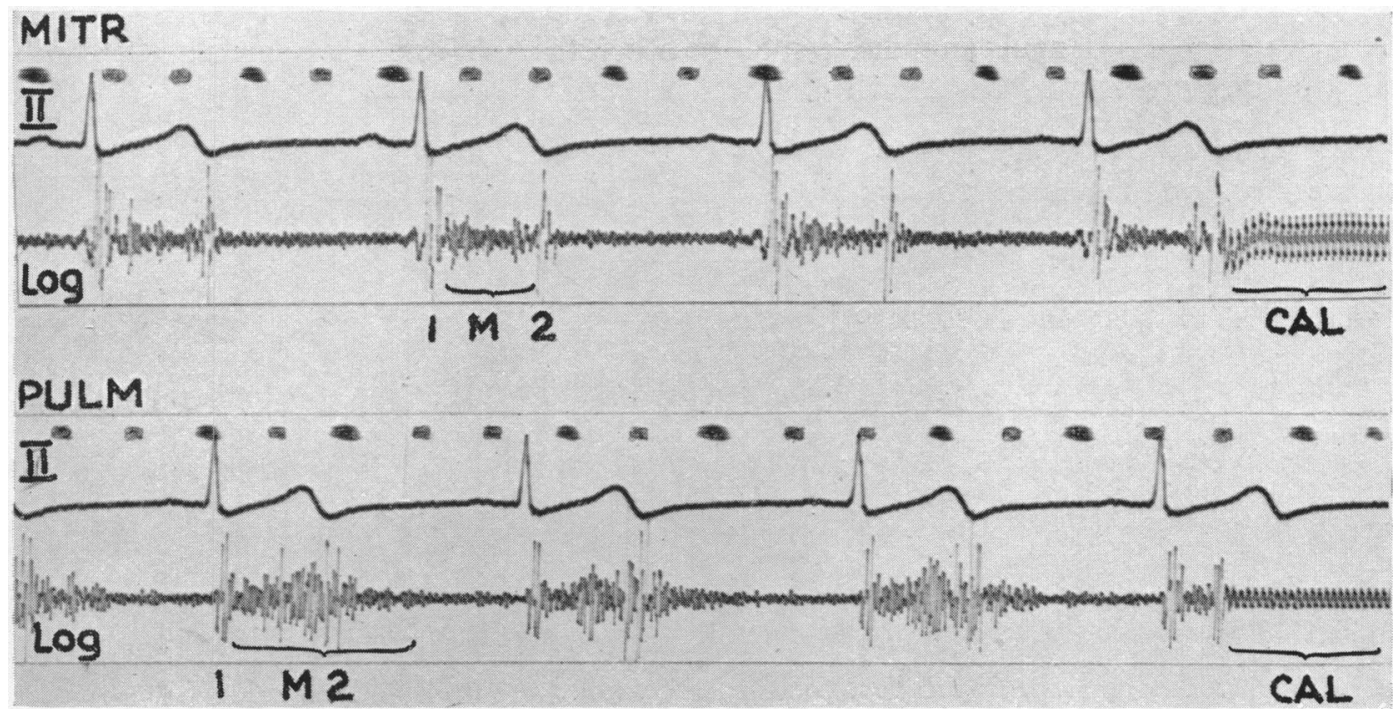

FIG. 3.-Calibrated phonocardiograms from a patient with patent ductus arteriosus. MITR, record from mitral area; PULM, record from pulmonary area; M, murmur. At the mitral area, in spite of high gain on the phonocardiograph, the murmur is not of great amplitude; at the pulmonary area the murmur is of much greater amplitude in spite of reduced gain on the instrument, shown by the small calibration signal.

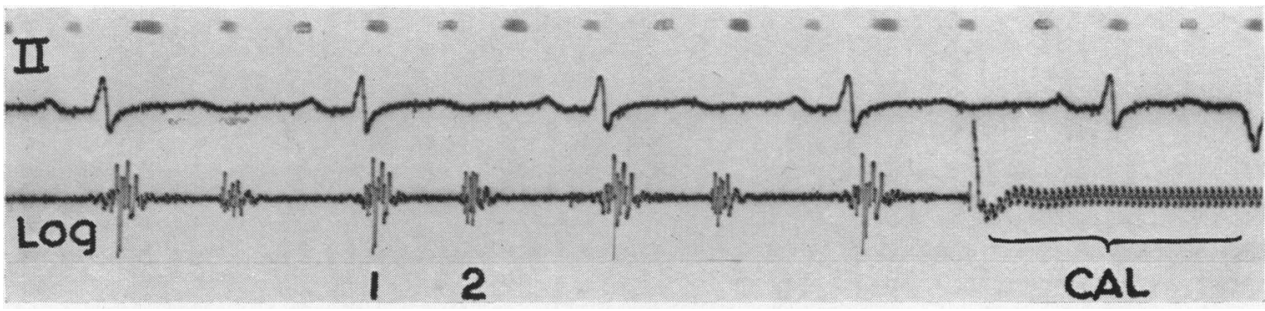

FIG. 4.-Calibrated phonocardiogram from the mitral area of a patient with mitral stenosis. By comparison with the small signal the sounds are seen to be loud; at this low gain a diastolic murmur, which was clearly audible, is not visible on the record. With the gain necessary to show the murmur the deflections corresponding to the heart sounds would be too large for their whole amplitude to be recorded.

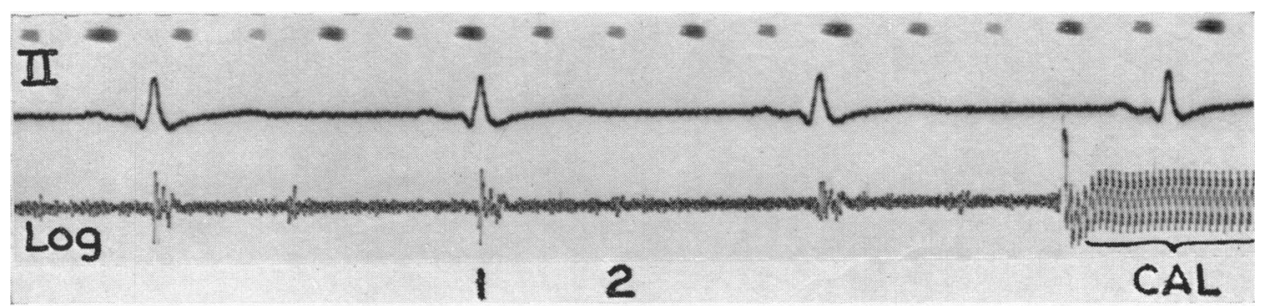

FIG. 5.-Calibrated phonocardiogram from the mitral area of a patient with pulmonary emphysema. The heart sounds are faint by contrast with the signal. 
above the threshold of audibility at this frequency, is recorded at the end of each phonocardiogram. The amplitude of this signal is an indication of the absolute loudness of the sounds at the point of application of the microphone to the chest wall.

We acknowledge our indebtedness to the Rankin Medical Research Fund of the University of Glasgow for defraying expenses and to Mr. J. Brown for technical assistance.

\section{REFERENCES}

Besterman, E. M. M., and Harrison, J. K. (1953). Brit. Heart J., 15, 130.

Campbell, F. W., Sloan, A. W., and Andrew, A. M. (1952). Brit. Heart J., 14, 271.

Counihan, T. B., Rappaport, M. B., and Sprague, H. B. (1951). Circulation, 4, 716.

Dunn, F. L., and Rahm, W. E. (1952). Amer. Heart J., 44, 95.

,,$--(1953 a)$. Amer. Heart J., 45, 519.

- - (1953b). Amer. Heart J., 46, 237.

Fletcher, H. (1948). Speech and Hearing. 7th ed., D. Van Nostrand Co., Inc., New York.

Mannheimer, E. (1940). Acta paediatr. Stockh., 28, Suppl. 2.

(1941). Amer. Heart J., 21, 151.

Olson, H. F., and Massa, F. (1939). Applied Acoustics. 2nd ed., Constable \& Co., London.

Rappaport, M. B., and Sprague, H. B. (1942). Amer. Heart J., 23, 591.

Wells, B. G., Rappaport, M. B., and Sprague, H. B. (1949). Amer. Heart J., 37, 586.

Williams, H. B., and Dodge, H. F. (1926). Arch. intern. Med., 38, 685.

Wood, A. (1940). Acoustics. Blackie \& Son, London and Glasgow. 